\section{Contexto familiar e alterações oclusais em pré-escolares no município de Salvador, Bahia, Brasil}

\author{
Influence of familiar context and \\ malocclusion in children aged $0-5$ years-old \\ in the city of Salvador, State of Bahia, Brazil
}

Thaís Regis Aranha Rossi 1

Laíra Sá Lopes 2

Maria Cristina Teixeira Cangussu 3

1-3 Departamento de Odontologia Social e Pediátrica. Faculdade de Odontologia. Universidade Federal da Bahia. Rua Araújo Pinho 62. Salvador, BA, Brasil. CEP: 41.110-150.

E-mail: cangussu@ufba.br

\begin{abstract}
Objectives: the purpose was to investigate the prevalence and the association between social and environmental variables and the children malocclusion.

Methods: the population for this cross-sectional study comprised 360 families (415 schoolchildren) resident in six areas where the Family Health Program is running in Salvador in the Brazilian State of Bahia. The dental examinations were carried out using criteria identifying the presence and type of malloclusion and a structured interview conducted with children's mothers or guardians. "Document A" and CAGE were used to report the social and environmental variables. Logistic regression (stepwise forward procedure, $p \leq 0.05$ ) analyses were performed.

Results: the prevalence of malocclusion was $34.5 \%$. Most families (77.2\%) lived in dwellings with thick partition walls. Exclusive breastfeeding rates were $64.8 \%$ and of these, $50.6 \%$ for the duration of the first seven months of life or more. $89.6 \%$ did not have the habit of digital suction, but the use of a pacifier was registered in 47\%. The multivariate analysis identified an association with malocclusion: the number of individuals aged under 14 years in the household $(O R=1.44 ; 95 \% C I: 1.02-2.26)$, digital sucking $(O R=1.75 ; 95 \% C I: 1.08-3.46)$ and the presence of alcoholism in the family $(O R=1.22 ; 95 \% C I$ : $1.04-2.02)$

Conclusions: access to oral health for these families can have a positive impact on oral health.
\end{abstract}

Key words Family relations, Child, preschool, Malocclusion

\section{Resumo}

Objetivos: avaliar a prevalência e a associação entre variáveis sócio-ambientais e a maloclusão infantil.

Métodos: estudo transversal, em crianças de 1-5 anos, residentes em seis áreas do Programa de Saúde da Família, em Salvador, Bahia, Brasil. Foram sorteadas 360 famílias pré-estratificadas, pelo cadastro das famílias das Unidade de Saúde da Família (USF) (415 pré-escolares). Após a identificação, foram realizadas visitas domiciliares onde foram entrevistados os pais/responsáveis e as crianças examinadas. Foram utilizados como instrumentos a ficha A - Sistema de Atenção Básica, e o exame oclusal - presença ou ausência de alteração $e$ tipo de desvio. A análise de regressão logística foi utilizada $(p<0,05)$.

Resultados: $34,5 \%$ das crianças apresentaram maloclusão; 77,2\% residiam em habitações com mais de quatro cômodos e $64,8 \%$ das crianças foi aleitada naturalmente e destas, 50,6\% por sete meses ou mais. Não tinham o hábito da sucção digital, 89,6\% das crianças, mas uso da chupeta foi registrado em $47 \%$. A análise de regressão logística identificou as associações: o número de individuos menores de 14 anos na residência $(O R=1,44$; IC95\%: 1,02 - 2,26), sucção digital $(O R=1,75$; IC95\%: 1,08-3,46) e ter adulto com uso abusivo de álcool $(O R=1,22$; IC95\%: 1,042,02).

Conclusões: ampliar o acesso à saúde oral pode refletir positivamente na saúde oral e qualidade de vida das crianças.

Palavras-chave Relações familiares, Pré-escolares, Maloclusão 


\section{Introdução}

A criança, ao nascer, é um indivíduo dependente do amparo e do cuidado da família. O recém-nascido inicia a sua adaptação ao meio através da assimilação, traduzida inicialmente pelo ato de sugar. ${ }^{1,2} \mathrm{O}$ reflexo da sucção conduz à discriminação e ao reconhecimento. O lactente não se contenta em sugar quando mama, sugando também no vazio, seus dedos e qualquer objeto. Para ele o mundo é essencialmente uma realidade a sugar.

Em relação à sucção não nutritiva, Freud ${ }^{3}$ descreve o prazer de chupar o dedo como uma satisfação autoerótica partida de uma zona erógena, a fase oral. Os hábitos de sucção trazem consequências importantes na morfologia do palato duro, alterações de posicionamentos dentais, movimentação da língua, com alterações musculares periorais e fonoarticulatórias, tendo maior risco de desenvolvimento de mordida aberta e distúrbios de motricidade oral.4-6

A sucção não nutritiva tem sido bastante associada ao estabelecimento de maloclusões, especialmente a sucção de chupeta ou de dedo.7,8 De acordo com a teoria psicanalítica, crianças que têm mais hábitos de sucção não nutritiva foram bebês que tiveram menos oportunidade de sugar. Já a teoria da aprendizagem afirma que bebês com considerável reforço dos hábitos orais tenderão mais tarde a adquiri-los de forma generalizados, mas não descreve vínculos emocionais associados aos hábitos de sucção. 9

Ambas tentam justificar o prolongamento que certas crianças apresentam na fase oral, caracterizada pela persistência e duração do hábito oral indesejável. Na odontologia, a persistência do hábito aliada ao surto do crescimento, à predisposição do indivíduo e, em certos casos, às condições nutricionais insatisfatórias e consequente deficiência da saúde, podem causar deformações morfológicas no infante, a exemplo das oclusopatias. 10

Relacionando os hábitos deletérios ao desenvolvimento, pesquisas realizadas no início do século $\mathrm{XX}$ já demonstravam que grupos de crianças que chupavam dedos tinham sido amamentados pela mãe com menos frequência e por períodos mais breves que um grupo de crianças que não tinham esse hábito. 9

Em 1981, Bolwby,11,12 aborda a relação mãecriança extrapolando a amamentação, e analisando estudos de observação direta, conclui que quando uma criança é privada dos cuidados maternos, o seu desenvolvimento tem como consequências retardados físicos, intelectual e social, e que podem se manifestar sintomas de doença física e mental. A figura da mãe em todos os níveis de renda é fundamental, pois é identificada como a responsável pela saúde da família. ${ }^{13}$

Assim sendo, as doenças maternas, ocorridas no período pré-escolar, mostram-se associadas ao déficit no desenvolvimento e ao acometimento de enfermidades nos filhos. ${ }^{14}$ Famílias com conflitos parentais e mães estressadas psicologicamente influenciam as condições de saúde das crianças. 15

Se o ambiente familiar não oferece amor, limites e atenção necessária para a criança, esta poderá apresentar dificuldades no convívio social, problemas no seu desenvolvimento e distúrbios psicológicos. ${ }^{16} \mathrm{~A}$ família é o lugar onde o indivíduo se constitui e desenvolve a sua subjetividade, sempre tendo com modelo de identificação seus pais. Desta forma, qualquer distúrbio presente no cuidador exercerá forte influência no comportamento da criança.

Em relação aos problemas orais, no Brasil, as crianças apresentam um dos mais altos índices de extrações dentárias prematuras, sem manutenção do espaço perdido. Destacam-se, ainda, as extensas lesões de cárie, que quando não tratadas também contribuem para a determinação de maloclusões, alterações que figuram na terceira posição na escala de prioridades e de problemas de saúde oral.10,17 Entretanto, são poucos os estudos sobre as alterações oclusais nesta faixa etária. $7,18,19$

Embora as informações epidemiológicas sejam resultantes da análise de estudos transversais, incluindo os possíveis vieses inerentes a essas pesquisas, reconhece-se que a prevalência e a severidade das oclusopatias têm aumentado nos últimos anos, especialmente o “apinhamento' dentário, tanto na dentição decídua como permanente. $18 \mathrm{O}$ último estudo realizado pelo Ministério da Saúde, em 2003, apresentou que $36,46 \%$ das crianças brasileiras com cinco anos tinham maloclusão, sem identificar, entretanto, as características das alterações em função do instrumento utilizado. 20

Estudos mostram que as oclusopatias são um fenômeno das "civilizações modernas", predominantemente urbanas. Sugerem também que são influenciadas por fatores do meio ambiente e comportamentais, como a alimentação menos consistente, infecções respiratórias, perda precoce de dentes decíduos e, principalmente, hábitos bucais deletérios. ${ }^{19}$

Umas das principais medidas de promoção de saúde, o aleitamento natural não só está relacionado com os aspectos nutricionais, como também preenche as necessidades emocionais do bebê, através do contato próximo estabelecido entre a mãe 
e o filho. ${ }^{7}$

Em função do exposto, o objetivo do presente estudo foi analisar a prevalência das oclusopatias em áreas cobertas pelo Programa de Saúde da Família.

\section{Métodos}

Realizou-se um estudo de prevalência analítico, na cidade de Salvador, Bahia, Brasil, em 2005, cuja população de referência constituiu-se de pré-escolares que residiam em seis áreas cobertas pelo Programa de Saúde da Família (PSF) do município.

A população de referência foi de crianças da primeira infância (até cinco anos) residentes em áreas cobertas pelo Programa de Agentes Comunitários de Saúde (PACS) e/ou PSF (30\% da população total do município). O cálculo do tamanho amostral foi realizado considerando-se a população finita definida pelo total de crianças residentes em áreas cobertas pelo PSF, prevalência de oclusopatias em crianças ao nível de $20 \%$ e $R R=2,0$ para associação entre contexto familiar e alteração de oclusão, um nível de confiança de $95 \%$ e um poder de $80 \%$ resultando $n=362$. A amostra foi préestratificada por Distrito Sanitário, utilizando como parâmetro de proporcionalidade o número de equipes de saúde bucal implantadas em cada distrito. Realizou-se, então, sorteio de seis Equipes de Saúde Bucal com possibilidade de substituição, em caso de recusa. A inclusão dos indivíduos no estudo se deu a partir do sorteio de 60 famílias que tinham crianças na faixa etária do estudo cadastradas nas Unidades pelo PACS.

Após a identificação da criança e prévia autorização da família, efetuaram-se visitas domiciliares pelas seis equipes previamente calibradas, uma em cada área. Foram excluídas as famílias em que não fosse possível a identificação da mãe ou responsável para responder a entrevista.

A coleta de dados compreendeu o exame clínico das crianças para avaliação da maloclusão na dentição decídua e a entrevista das suas respectivas mães, através da aplicação de questionário estruturado. A calibração interexaminador foi previamente realizada com os seis examinadores responsáveis pelo exame. O percentual geral de concordância de diaganóstico foi de $85,1 \%$ e após o cálculo do kappa na concordância 2 a 2 , o valor mínimo obtido foi de 0,63 e o máximo 0,69 . O exame clínico foi realizado no domicílio, em ambiente externo, com iluminação natural, sem secagem ou escovação prévia dos dentes, utilizando-se abaixador de língua, estando a criança em posição sentada. A presença de oclusopatia foi registrada utilizando o indicador preconizado por Frazão et al.18 que identificava ausência ou presença de alteração oclusal, e caso esta estivesse presente, o tipo de alteração, incluindo presença de mordida aberta anterior, sobremordida, mordida cruzada, em topo e apinhamento. 17-19 Em seguida, ou em data agendada, foram realizadas as entrevistas com as mães, buscando-se resguardar a privacidade necessária. O instrumento elaborado estava dividido em seções que envolviam: a) identificação, dados socioeconômicos, demográficos e condições gerais de saúde materna/Ficha A do Sistema de Atenção Básica do Ministério da Salúde (1998); b) dados relativos à saúde oral na família e da criança/critérios (variáveis comportamentais de hábitos de higiene, sucção nutritiva - aleitamento materno, uso de mamadeira e não nutritiva - sucção de dedo e chupeta, dieta e acesso a assistência à saúde) e c) a escala CAGE adaptada - teste de triagem autoinformativo para alcoolismo que compreende quatro questões, sendo considerado positivo quando o resultado apresenta duas ou mais respostas afirmativas. 21

Procedeu-se a análise descritiva das variáveis de interesse de acordo com a exposição. Para identificar as variáveis associadas à ocorrência de oclusopatias em crianças de 0 e 5 anos, odds ratios (OR) foram estimados por meio de regressão logística não-condicional e a inferência estatística baseou-se em intervalos de confiança a $95 \%$, calculados pelo Wald Test. A seleção das variáveis para modelagem baseou-se na literatura e nos resultados da análise bivariada através do teste do qui-quadrado com um nível de significância de 5\%. Para a criação do banco de dados, utilizou-se o Epi-Info, versão 6.04 e a análise foi realizada no Minitab versão 14 .

O protocolo desta pesquisa foi aprovado pelo Comitê de Ética em Pesquisa da Maternidade Climério de Oliveira da Universidade Federal da Bahia, em 10 de janeiro de 2005 (Registro CEP: 026-04/CEP-COM).

\section{Resultados}

Foram examinadas 415 crianças, provenientes de 360 famílias, das quais $43 \%$ pertenciam ao sexo feminino e $57 \%$ ao masculino, a maior parte da amostra era constituída de indivíduos pardos, $63 \%$. A prevalência de maloclusão foi de $34,5 \%$ e o tipo mais frequente foi a mordida aberta anterior (Figura $1)$.

Considerando-se a caracterização da população de estudo de acordo com a exposição, observou-se alto grau de homogeneidade entre expostos e não expostos, quando consideradas as variáveis fami- 
Figura 1

Tipo de oclusopatia mais prevalente, pré-escolares de área cobertas pelo Programa Saúde da Família. Salvador, Bahia, 2005 ( $n=415)$.

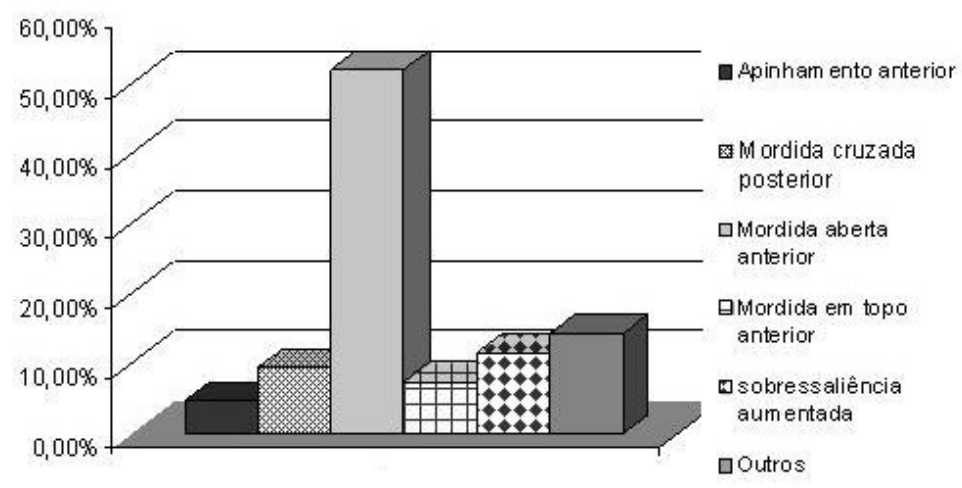

Tabela 1

Variáveis sócio-demográficas associadas à ocorrência de alterações oclusais em pré-escolares de áreas cobertas pelo Programa Saúde da Família. Salvador, Bahia, 2005 ( $n=415)$.

\begin{tabular}{|c|c|c|c|c|c|}
\hline \multirow[t]{2}{*}{ Variáveis } & \multicolumn{2}{|c|}{ Maloclusão (n=168) } & \multicolumn{2}{|c|}{ Oclusão normal $(n=247)$} & \multirow[t]{2}{*}{ Valor $p$} \\
\hline & $\mathrm{n}$ & $\%$ & $\mathrm{n}$ & $\%$ & \\
\hline \multicolumn{6}{|c|}{ Indivíduos >15 anos } \\
\hline Até 4 & 127 & 75,59 & 192 & 77,73 & 0,612 \\
\hline 5 ou mais & 41 & 24,40 & 55 & 22,26 & \\
\hline \multicolumn{6}{|c|}{ Analfabetos $>15$ anos } \\
\hline Nenhum & 114 & 67,86 & 169 & 68,69 & 0,856 \\
\hline 1 ou mais & 54 & 32,14 & 77 & 31,30 & \\
\hline \multicolumn{6}{|c|}{ Indivíduos $<14$ anos } \\
\hline Até 2 & 93 & 55,35 & 153 & 61,94 & 0,180 \\
\hline Mais de 3 & 75 & 44,64 & 94 & 31,30 & \\
\hline \multicolumn{6}{|c|}{ Analfabetos $<14$ anos } \\
\hline Até 2 & 155 & 92,26 & 237 & 96,34 & 0,109 \\
\hline Mais de 2 & 12 & 7,14 & 9 & 3,65 & \\
\hline \multicolumn{6}{|c|}{ Uso abusivo de álcool } \\
\hline Não & 132 & 78,57 & 187 & 75,71 & 0,497 \\
\hline $\operatorname{Sim}$ & 36 & 21,43 & 60 & 24,29 & \\
\hline
\end{tabular}

liares. A maioria das famílias era composta por menos de quatro indivíduos. Conforme os dados apresentados na Tabela 1 , houve predomínio de alfabetizados, com famílias de até dois indivíduos menores de 14 anos e que não apresentavam uso abusivo de álcool, mensurado pela escala CAGE.

Os dados da Tabela 2 apontam que a maior parte das famílias não possuía plano de saúde médico $(75,2 \%)$ e que muitos indivíduos $(77,2 \%)$ residiam em habitações com mais de quatro cômodos. O meio de transporte mais utilizado foi o ônibus $(93,8 \%)$ e quase a totalidade da amostra residia em casa de tijolo/adobe $(95,64 \%)$.

Do total, $43,6 \%$ das crianças realizavam uma escovação por dia ou não escovavam e $64,2 \%$ consumiam doces mais de três vezes ao dia. A maioria foi amamentada durante o período noturno; $64,8 \%$ das crianças foram aleitadas naturalmente e 
Tabela 2

Variáveis ambientais associadas à ocorrência de alterações oclusais em pré-escolares de áreas cobertas pelo Programa Saúde da Família. Salvador, Bahia, 2005. $(n=415)$.

\begin{tabular}{|c|c|c|c|c|c|}
\hline \multirow[t]{2}{*}{ Variáveis } & \multicolumn{2}{|c|}{ Maloclusão (n=168) } & \multicolumn{2}{|c|}{ Oclusão normal $(n=247)$} & \multirow[t]{2}{*}{ Valor $p$} \\
\hline & $\mathrm{n}$ & $\%$ & $\mathrm{n}$ & $\%$ & \\
\hline \multicolumn{6}{|l|}{ Tipo de Casa } \\
\hline Tijolo / Adobe & 158 & 94,05 & 237 & 96,73 & 0,188 \\
\hline Outros & 10 & 5,95 & 8 & 3,26 & \\
\hline \multicolumn{6}{|c|}{ Número de cômodos } \\
\hline Até 3 & 33 & 20,00 & 61 & 24,69 & 0,570 \\
\hline Mais de 4 & 132 & 80,00 & 186 & 75,30 & \\
\hline \multicolumn{6}{|l|}{ Transporte } \\
\hline Ônibus & 142 & 92,20 & 192 & 86,87 & 0,269 \\
\hline Outros & 12 & 7,78 & 10 & 13,11 & \\
\hline \multicolumn{6}{|c|}{ Plano de saúde médico } \\
\hline $\operatorname{sim}$ & 48 & 28,57 & 55 & 22,27 & 0,144 \\
\hline Não & 120 & 71,43 & 192 & 77,73 & \\
\hline
\end{tabular}

\section{Tabela 3}

Variáveis individuais associadas à ocorrência de alterações oclusais em pré-escolares de áreas cobertas pelo Programa Saúde da Família. Salvador, Bahia, 2005. ( $n=415)$.

\begin{tabular}{|c|c|c|c|c|c|}
\hline \multirow[t]{2}{*}{ Variáveis } & \multicolumn{2}{|c|}{ Maloclusão (n=168) } & \multicolumn{2}{|c|}{ Oclusão normal $(n=247)$} & \multirow[t]{2}{*}{ Valor $p$} \\
\hline & $\mathrm{n}$ & $\%$ & $\mathrm{n}$ & $\%$ & \\
\hline Sexo & & & & & 0,59 \\
\hline Masculino & 86 & 51,19 & 133 & 53,85 & \\
\hline Feminino & 82 & 48,81 & 114 & 46,15 & \\
\hline Escolaridade & & & & & 0,81 \\
\hline Não analfabeto & 10 & 8,25 & 17 & 7,83 & \\
\hline Analfabeto & 111 & 91,73 & 200 & 92,16 & \\
\hline Utilização medicamento & & & & & 0,00 \\
\hline $\operatorname{sim}$ & 17 & 22,66 & 118 & 85,50 & \\
\hline Não & 58 & 71,33 & 20 & 14,49 & \\
\hline Utilização Serviço Saúde & & & & & 0,00 \\
\hline Sim & 124 & 74,25 & 61 & 25,41 & \\
\hline Não & 43 & 25,74 & 179 & 74,58 & \\
\hline Escovações por dia & & & & & 0,00 \\
\hline Nenhuma ou 1 & 6 & 3,57 & 108 & 43,72 & \\
\hline Mais de 2 & 162 & 96,43 & 139 & 56,28 & \\
\hline Consumo de Doces & & & & & 0,00 \\
\hline$<2$ vezes ao dia & 40 & 23,81 & 108 & 43,72 & \\
\hline$>3$ vezes ao dia & 127 & 75,59 & 139 & 56,28 & \\
\hline Amamentação noturna & & & & & 0,16 \\
\hline Não & 50 & 30,48 & 59 & 24,18 & \\
\hline $\operatorname{Sim}$ & 114 & 69,51 & 185 & 75,81 & \\
\hline Aleitamento natural & & & & & 0,01 \\
\hline Sim & 96 & 57,14 & 173 & 70,04 & \\
\hline Não & 67 & 39,88 & 72 & 29,15 & \\
\hline
\end{tabular}


conclusão

Tabela 3

Variáveis Individuais associadas à ocorrência de alterações oclusais em pré-escolares de áreas cobertas pelo Programa Saúde da Família. Salvador, Bahia, 2005. ( $n=415)$.

\begin{tabular}{|c|c|c|c|c|c|}
\hline \multirow[t]{2}{*}{ Variáveis } & \multicolumn{2}{|c|}{ Maloclusão (n=168) } & \multicolumn{2}{|c|}{ Oclusão normal ( $n=247)$} & \multirow[t]{2}{*}{ Valor $p$} \\
\hline & $\mathrm{n}$ & $\%$ & $\mathrm{n}$ & $\%$ & \\
\hline Tempo Aleitamento natural & & & & & 0,00 \\
\hline Menos de sete meses & 94 & 55,95 & 173 & 70,04 & \\
\hline Mais de oito meses & 74 & 44,04 & 72 & 29,15 & \\
\hline Sucção digital & & & & & 0,04 \\
\hline Sim & 21 & 12,50 & 17 & 6,88 & \\
\hline Não & 143 & 85,12 & 229 & 92,71 & \\
\hline Tempo de sucção digital & & & & & 0,09 \\
\hline Até 12 meses & 7 & 31,81 & 4 & 25,00 & \\
\hline Mais de 12 meses & 15 & 68,18 & 12 & 75,00 & \\
\hline Uso da chupeta & & & & & 0,00 \\
\hline Sim & 103 & 61,31 & 92 & 37,25 & \\
\hline Não & 62 & 36,90 & 154 & 62,35 & \\
\hline Tempo da chupeta & & & & & 0,00 \\
\hline Até 36 meses & 77 & 74,75 & 95 & 94,05 & \\
\hline Mais de 36 meses & 26 & 25,24 & 6 & 5,94 & \\
\hline Frequência creche & & & & & 0,11 \\
\hline Sim & 50 & 29,76 & 91 & 37,39 & \\
\hline Não & 118 & 70,24 & 153 & 62,60 & \\
\hline
\end{tabular}

Tabela 4

Resultado final da regressão logística das variáveis associadas à ocorrência de alterações oclusais em préescolares de áreas cobertas pelo Programa Saúde da Família. Salvador, Bahia, 2005. $(n=415)$.

\begin{tabular}{|c|c|c|}
\hline Variáveis & $\begin{array}{l}\text { Odds Ratio } \\
\text { ajustado }\end{array}$ & IC95\% \\
\hline $\begin{array}{l}\text { Não utilizar } \\
\text { serviços de saúde }\end{array}$ & 0,92 & $0,56-1,49$ \\
\hline $\begin{array}{l}\text { Uma ou menos } \\
\text { escovações /dia }\end{array}$ & 1,13 & $0,73-1,76$ \\
\hline Alto consumo de doces & 1,21 & $0,75-1,95$ \\
\hline $\begin{array}{l}\text { >3 indivíduos com } \\
\text { menos de } 14 \text { anos }\end{array}$ & 1,44 & $1,02-2,26$ * \\
\hline Aleitamento natural & 0,84 & $0,19-1,85$ \\
\hline Hábito de sucção digital & 1,75 & $1,08-3,46$ * \\
\hline Uso de chupeta & 0,99 & $0,99-1,00$ \\
\hline $\begin{array}{l}\text { Uso abusivo de álcool } \\
\text { na família }\end{array}$ & 1,22 & $1,04-2,02$ * \\
\hline
\end{tabular}

Máxima verossimilhança $=-250,128 ; p=0,001$; * variáveis com significância estatística. destas, $50,6 \%$ por sete meses ou mais. $89,6 \%$ das crianças não tinham o hábito da sucção digital, mas o uso da chupeta foi registrado em $47 \%$ (Tabela 3 ).

Na regressão logística de caráter exploratório (Tabela 4) foram obtidos como potenciais indicadores de risco: mais de três indivíduos menores de 14 anos na residência ( $\mathrm{OR}=1,44$; IC95\%: $1,02-$ $2,26)$, hábito de sucção digital ( $\mathrm{OR}=1,75$; IC95\%: 1,08-3,46) e adulto com histórico de uso abusivo de álcool na residência (OR=1,22; IC95\%: 1,04-2,02).

\section{Discussão}

A partir da análise dos achados, observou-se associação entre presença de oclusopatia e não ter sido amamentado naturalmente, e tempo de aleitamento natural menor ou igual a sete meses (Tabela 4 ). $\mathrm{O}$ aleitamento natural não só está relacionado com os aspectos nutricionais, como também preenche as necessidades emocionais do bebê, através do contato próximo estabelecido entre a mãe e o filho. ${ }^{7}$ Ao sugar o seio materno, a criança estabelece o padrão adequado de respiração nasal e postura correta da língua. Ocorre a estimulação dos músculos durante a 
sucção do seio materno, aumento do tônus e promoção da postura correta para futuramente exercer a função de mastigação. Além desses aspectos, destaca-se que o desenvolvimento motororal reflete no desenvolvimento craniofacial, no crescimento ósseo e na dentição. ${ }^{4-6}$

Além de prevenir o desenvolvimento de maloclusões, a literatura mostra que há associação negativa entre a amamentação e a instalação de hábitos de sucção não nutritiva. Commerford 22 concluiu em seu estudo que $92 \%$ das crianças que receberam alimentação no seio materno como forma exclusiva de alimentação nos primeiros seis meses de vida não apresentaram estes hábitos. Também Serra-Negra et al., 7 em 1996, encontraram uma taxa de 86,1\% de aleitamento natural e a ausência dos hábitos de sucção por mais de seis meses.

O uso da chupeta e seu tempo de utilização foram variáveis relevantes no nosso estudo $(p<0,05)$. A sucção digital foi apontada como potencial fator associado às oclusopatias (OR=1,75; IC95\%: 1,08$3,46)$. A relação entre os hábitos bucais deletérios e as alterações oclusais é bastante difundida na literatura. 7,10,17 Emmerich et al.,6 em 2004, avaliaram crianças com três anos, concluindo que hábitos orais de sucção de chupeta ou de dedo foram capazes de causar uma maior incidência de anomalias na oclusão dentária decídua. O risco relativo da associação entre mordida aberta anterior, o tipo de maloclusão mais prevalente no nosso estudo, e o hábito de chupar dedo foi de 12,4 no estudo de Paunio et al.23 Os hábitos de sucção trazem consequências importantes na morfologia do palato duro, alterações de posicionamentos dentais, movimentação da língua, com alterações musculares periorais e fonoarticulatórias, tendo maior risco de desenvolvimento de mordida aberta e distúrbios de motricidade oral. $4-6$

O número de escovações por dia, o consumo de doces, a maior utilização dos serviços de saúde pelas crianças que apresentavam maloclusão e não utilização de medicamentos (Tabela $3 ; p<0,05$ ) nos remetem às variáveis relacionadas à cárie e, devido aos sintomas secundários dessa doença, a procura pelos serviços de saúde. As crianças brasileiras apresentam um dos mais altos índices de extrações dentárias prematuras, sem manutenção do espaço perdido. Lesões de cárie extensas não tratadas podem ser fatores agravantes na determinação de más oclusões. ${ }^{10}$ Tomita et al. 17 já destacavam que o acesso a bens e serviços de saúde oral circunscrevem- se aos grupos sociais mais favorecidos, de forma que apenas $5 \%$ da população têm acesso regular a esses serviços, estimando-se que de $15 \%$ a $17 \%$ o consumam de forma irregular.

$\mathrm{O}$ número de indivíduos menores de 14 anos na residência ( $O R=1,44$; IC95\% 1,02 - 2,26) foi um indicador potencial de risco no nosso estudo. O estudo de Martins et al.,8 realizado no Brasil, revelou que casas com famílias numerosas e a presença de mais de quatro irmãos na mesma residência estiveram associados à qualidade do ambiente. Outros estudos, como o de Martins et al. ${ }^{8}$ e de Souza et al., ${ }^{21}$ consideraram essa variável como indicador de risco para a qualidade do ambiente, pois famílias numerosas tendem a ser menos estimuladoras. Consideramos que mais de três indivíduos menores que 14 anos na residência, num contexto de pobreza, predominante no nosso estudo, potencializa o risco 17 e conduz à redução na atenção dada a cada criança e consequente maior predisposição ao hábitos de sucção não nutritiva compensatórios.

O alcoolismo, por sua vez, está comumente associado com altos níveis de violência e baixos níveis de afeto no contexto familiar11,14,24 e piores condições de saúde oral. No presente estudo, cuidadores com escala CAGE positiva para duas ou mais perguntas representou ser um indicador de risco potencial para o desenvolvimento de oclusopatias nas crianças. A escala CAGE é um instrumento de validação que pode ser utilizado para identificar indivíduos dependentes etílicos. 25 Essa escala foi criada para identificar os usuários abusivos de álcool, relatos de problemas secundários devido ao uso de álcool, e usuários em risco, sem relatos de problemas devido ao uso do álcool, mas que bebem acima do limite seguro.26,27 Ressalta-se, contudo, que a escala CAGE pode produzir um número excessivo de falsos positivos por ser um instrumento com poucas perguntas, sugerida pela Organização Mundial da Saúde (OMS), constando apenas de quatro perguntas. 28

Autores $23,28,29$ alertam que o consumo excessivo de álcool pode ter consequências diretas no cuidado de pré-escolares. Na maioria das vezes, os filhos sofrem com uma interação familiar negativa e um empobrecimento na solução de problemas, uma vez que essas famílias são consideradas desorganizadas e disfuncionais. Filhos de dependentes químicos apresentam risco aumentado para transtornos psiquiátricos, desenvolvimento de problemas físicoemocionais e dificuldades escolares. ${ }^{28}$ Pode-se dizer que há consequências desfavoráveis, tanto para a saúde física quanto para o desenvolvimento psicos- 
social de crianças, quando há ocorrência de alcoolismo na família. Em idade pré-escolar, dada a grande vulnerabilidade das crianças, o sofrimento é maior com as disfunções familiares causadas pelo uso abusivo do álcool.21

Apesar de os resultados acima permitirem identificar padrões de comportamento por meio do conhecimento do contexto familiar de uma determinada população, através dos quais é possível delinear estratégias para enfrentar esses problemas, é importante ressaltar os limites dos mesmos. O desenho do estudo adotado tem os seus limites em relação ao viés de memória em diversas informações comportamentais, principalmente em crianças mais velhas, como o aleitamento, sucção, dieta, entre outros. Além disso, a própria entrevista poderia se constituir um viés positivo para a melhor condição esperada. Em relação às alterações oclusais, também é reconhecido o retorno a normalidade de forma espontânea após a suspensão de hábitos deletérios. Sendo assim, é possível que em idades mais avançadas a prevalência encontrada seja inferior a registrada neste estudo.

\section{Referências}

1. Piaget J, Inhelder B. A psicologia da criança (1989). 14. ed. Rio de Janeiro: Bertrand Brasil; 1995.

2. Flavell JH, Piaget J. A psicologia do desenvolvimento de Jean Piaget. 5. ed. São Paulo: Pioneira; 1996.

3. Freud S. A general introduction to psychoanalysis. New York: Permabooks; 1935.

4. Moresca CA, Feres NA. Hábitos viciosos bucais. In: Petrelli E. Ortodontia para fonoaudiologia. São Paulo: Lovise; 1994. p. 57-98.

5. Neiva FCB, Cattoni DM, Ramos JLA, Isler H. Desmame precoce: implicações para o desenvolvimento motor-oral. J Pediatr. (Rio J). 2003; 79: 7-12.

6. Emmerich A, Fonseca L, Elias AM, Medeiros UV. The relationship between oral habits, oronasopharyngeal alterations, and malocclusion in preschool children in Vitória, Espírito Santo, Brazil. Cad Saúde Pública. 2004; 20: 68997.

7. Serra-Negra JMC, Pordeus IA, Rocha JR JF. Study of the relationship between infant feeding methods, oral habits, and malocclusion. Rev Odontol Univ São Paulo. 1997; 13: 251-6.

8. Martins MFD, Costa JSD, Saforcada ET, Cunha MDC. Quality of the environment and associated factors: a pediatric study in Pelotas, Rio Grande do Sul, Brazil. Cad Saúde Pública. 2004; 20: 710-8.

9. Carmichael L. Manual de psicologia da criança: o primeiro ano de vida e as experiências iniciais. São Paulo: EPU, EDUSP; 1975. 329 p.
É importante ressaltar que os dados não são representativos para o município, dada as características da amostra, mas permitem inferir que programas vinculados à saúde infantil devem estar voltados para o ambiente da criança, pois é nele que ela se estrutura como um ser individual e social, capaz de interagir com outros níveis de amplitude e de construir espaços entre os setores sociais envolvidos no processo de construção de uma vida saudável. 8

Ressalta-se que poucos são os estudos que avançam no sentido de compreender a dinâmica familiar e as condições de saúde oral e o seu impacto na qualidade de vida.

\section{Conclusões}

Ampliar o acesso à informação e atenção à saúde oral para estas famílias é fundamental para que o ambiente familiar possa refletir positivamente na saúde oral e qualidade de vida das crianças.

10. Souza DFRK de, Valle MAS do, Pacheco MCT. Clinical relationship among suction oral habits, malocclusion, infant feeding and mother's previous knowledge. Rev Dent Press Ortodon Ortop Facial. 2006; 11: 81-90.

11. Bowlby J. Formação e rompimento dos laços afetivos. São Paulo: Martins Fontes; 1997.

12. Bowlby J. Separação: angústia e raiva. 4. ed São Paulo: Martins Fontes; 2004.

13. Martin VB, Angelo M. Family organization regarding children care: mothers perception in a low income community. Rev Latino-Am Enfermagem.1999; 7: 89-95.

14. Falcone VM. Disfunção familiar e baixa estatura em escolares da rede pública de ensino de São Paulo: estudo de caso-controle. [dissertação]. São Paulo: Curso de Pós-graduação em Nutrição da Escola Paulista de Medicina; 2001.

15. Drotar D. Relating parent and family functioning to the psychological adjustment of children with chronic health conditions: what have we learned? What do we need to know? J Pediatr Psychol. 1997; 22: 329-44.

16. Kalina E. Drogadição hoje: indivíduo, família e sociedade. Porto Alegre: Artmed; 1999. 232 p.

17. Tomita NE, Sheiham A, Bijella VT, Franco LJ. Relação entre determinantes socioeconômicos e hábitos bucais de risco para maloclusões em pré-escolares. Pesq Odontol Bras. 2000; 14: 169-75.

18. Frazão P, NarvaiI PC, LatorreE MRDO, Castellanos RA. Prevalência de oclusopatia na dentição decídua e permanente de crianças na cidade de São Paulo, Brasil, 1996. Cad Saúde Pública. 2002; 18: 1197-205. 
19. Peres KG, Traebert ESA, Marcenes W. Differences between normative criteria and self-perception in the assessment of malocclusion. Rev Saúde Pública. 2002; 36: 230-6.

20. Brasil. Ministério da Saúde, Secretaria de Atenção à Saúde, Departamento de Atenção Básica. Projeto SB Brasil 2003. Condições de saúde bucal da população brasileira, 2002 2003: resultados principais. Brasília, DF; 2004.

21. Souza MAA, Vianna MIP, Cangussu MCT. Family dysfunction related to maternal depression and/or alcoholism in the family and dental caries in children from two to three years old. Rev Bras Saúde Matern Infant. 2006; 6: 309-17.

22. Commerford M. Sucking habits in the breast-fed versus non breast-fed child. J Res Orofacial Muscle Imbal. 1977; 88: $18-9$.

23. Paunio P, RautavaP, Sillanpaa M. The finnish family competence study: the effects of living conditions on sucking habits in 3-year-old Finnish children and the association between these habits and dental occlusion. Acta Odontol Scand. 1993; 51: 23-9.

24. Carvalho IMM, Almeida PH. Família e proteção social. São Paulo Perspec. 2003; 17: 109-22.
25. Pokorny AD, Miller BA, Kaplan HB. The brief MAST: a shortened version of the Michigan Alcoholism Screening Test. Am J Psychiatr. 1972; 129: 342-5.

26. Malet L, Schwan R, Boussiron D, Aublet- Cuvelier B, Llorca PM. Validity of the CAGE questionnaire in hospital. Europ Psychiatr. 2005; 20: 484-9.

27. Mackenzie DM, Langa A, Brown TM. Identifying hazardous or harmful alcohol use in medical admissions: a comparison of AUDIT, CAGE and brief MAST. Alcohol Alcohol. 1996; 31: 591-9.

28. Figlie N, Fontes A, Moraes E, Payá R. Children of addicted parents with bio-psychosocial risk factors: do they need a special care? Rev Psiquiatr Clin. 2004; 31: 53-62.

29. Andraca I, Pino P, Parra A, Rivera F, Castilo M. Risk factors for psychomotor development among infants born under optimal biological conditions. Rev Saúde Pública. 1998; 32: 138-47.

Recebido em 28 de dezembro de 2008

Versão final apresentada em 9 de março de 2009

Aprovado em 13 de abril de 2009 